\title{
Bremsung und Absorption von Neutronen in Beton ${ }^{1}$
}

\author{
Von Peter Jensen ${ }^{2}$ und Oskar Ritter ${ }^{3}$ \\ Aus dem Institut für Physik am Kaiser-Wilhelm-Institut für medizinische Forschung, Heidelberg \\ (Z. Naturforschg. 2a, 376-384 [1947]; eingegangen am 17. April 1947)
}

\begin{abstract}
Die räumliche Verteilung von Neutronen verschiedener Energien im Innern eines großen Betonkörpers und der Neutronenstrom durch eine Betonwand variabler Dicke werden gemessen. Die aus den Messungen abgeleiteten Werte der Bremslänge und „Abklinglänge" für Neutronen werden mit berechneten Werten verglichen. Zur Berechnung der Abklinglänge wird eine neue Näherungsmethode benutzt, die es auch erlaubt, die Dicke von Neutronenschutzwänden zu berechnen. Eine Zusammenfassung der Ergebnisse befindet sich am Schluß der Arbeit.
\end{abstract}

$\mathrm{D}$ ie Ausbreitung der Neutronen in ausgedehnten materieerfüllten Räumen wurde erstmals von Fermi und Mitarbeitern ${ }^{4}$ um 1935 systematisch untersucht. In der folgenden Zeit nahm das Interesse an diesem Gegenstand etwas ab und wuchs erst wieder in den letzten Jahren im $\mathrm{Zu}$ sammenhang mit der Entwicklung der neuen starken Neutronenquellen. Von den zahlreichen einschlägigen Untersuchungen ist nur wenig veröffentlicht worden, in Deutschland zwei theoretische Arbeiten von $\mathrm{B}_{\text {oth }} \mathrm{e}^{5}$ und Flügge ${ }^{6}$ über die räumliche Verteilung abgebremster Neutronen und eine experimentelle Arbeit von $\mathrm{Jensen}^{7}$ über die Bremsung von Neutronen in Kohlenstoff und schwerem Wasser.

Die vorliegende Arbeit ist zum Teil eine Fortsetzung von ${ }^{7}$ und handelt von der Neutronenausbreitung in Beton. Dieses Material wurde untersucht, weil es als Baustoff für Neutronenschutzwände interessiert und weil es in so großer Menge verfügbar war, daß die Neutronenverteilung darin auch bis zu großen Quellabständen praktisch ohne Randstörungen gemessen werden konnte. Im einzelnen wurden gemessen: die Bremslänge für Ra+Be-Neutronen, die „Abklinglänge“ für thermische Neutronen, für Indium-Resonanzneutronen und für schnelle Neutronen, die die $\operatorname{Al}(n, p)$ Reaktion auslösen, endlich die Zahl der durch eine Betonwand hindurchtretenden Neutronen in Abhängigkeit von der Wandstärke.

Im theoretischen Teil der Arbeit wird u. a. das früher angegebene halbempirische Verfahren ${ }^{7}$ zur

1 Diese Arbeit wurde im November 1944 beendet. Die Veröffentlichung verzögerte sich aus äußeren Gründen.

2 Jetzt am Physikalischen Institut der Universität Freiburg i. Br.
Berechnung von Bremslängen auf den Fall des Betons angewandt. Ferner wird eine einfache Beziehung zwischen der Abklinglänge und der Bremslänge abgeleitet, die sich daraus ergibt, daß die Neutronenverlangsamung als eine Diffusion schneller Neutronen mit Absorption aufgefaßt wird, wobei das „Thermischwerden“ die Rolle der Absorption spielt. Damit wird es möglich, Abklinglängen und die Dicke von Neutronenschutzwänden näherungsweise $\mathrm{zu}$ berechnen.

1. Messungen und Meßergebnisse

a) Di e Bremslänge. Zur Bestimmung der Bremslänge $B$ wurde das Fermische Verfahren angewandt, d.h. es wurde die Dichte $\varrho$ von fastthermischen Resonanzneutronen als Funktion des Quellabstandes $r$ in einem hinreichend großen Betonvolumen gemessen und $B$ durch Integration gewonnen:

$$
B^{2}=\overline{r^{2}}=\int_{0}^{\infty} \varrho r^{4} d r / \int_{0}^{\infty} \varrho r^{2} d r .
$$

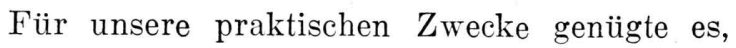
einen Betonkörper von $1 \times 1 \times 2 \mathrm{~m}$ Größe zu verwenden, auf dessen Längsachse die Neutronendichte bei Abständen $>50 \mathrm{~cm}$ von den Quadratflächen gemessen wurde. Die Neutronenquelle (500 mg Ra $+\mathrm{Be}$ ) befand sich am einen Ende der $1 \mathrm{~m}$ langen Meßstrecke, also allseitig mindestens

3 Jetzt am Physikalischen Institut der Universität Heidelberg.

4 E. A maldi u. E. Fermi, Physic. Rev. 50, 899 [1936]; dort weitere Literatur.

5 W. B o th e, Z. Physik 122, 648 [1944].

• S. F l ü g g e, Physik. Z. 44, 445 [1943].

7 P. Jensen, Z. Physik 122, 756 [1944]. 
$50 \mathrm{~cm}$ vom Rande entfernt. In Abschn. $2 \mathrm{~g}$ wird gezeigt, daß die so erhaltene Dichteverteilung von der für den unbegrenzten Körper zu erwartenden nur wenig abweichen konnte. Der Betonkörper wurde aus Platten von $50 \times 50 \times 10 \mathrm{~cm}$ Größe aufgebaut. Die Dichte des Betons betrug $2,26 \mathrm{~g} / \mathrm{cm}^{3}$, $1 \mathrm{~m}^{3}$ enthielt ca. $340 \mathrm{~kg}$ Zement und 1700 bis $1800 \mathrm{~kg}$ Kiessand. Der Wassergehalt des abgebundenen Betons kann nicht sicher angegeben werden, er dürfte zwischen 5 und 10 Gewichtsprozent liegen ${ }^{8}$.

Die Dichte der fast-thermischen Neutronen wurde mit Indium-Sonden bestimmt, die in Cadmium eingeschlossen waren. Die 57-min-Aktivität des In, dessen Resonanzenergie ca. $1 \mathrm{eV}$ beträgt, wurde mit dem Zählrohr gemessen.

In Abb. 1 ist die so erhaltene relative Dichte $\varrho$ der In-Resonanzneutronen, multipliziert mit dem Quadrat des Quellabstandes $r$, als Funktion von $r$ aufgetragen (Kurve $D$ ). Aus dieser Kurve, sowie aus einer entsprechenden für $\varrho r^{4}$, wurde die Bremslänge $B$ gemäß Gl. (1) durch Planimetrieren gewonnen. Die Teilintegrale von $r=110$ bis $r=\infty$ wurden rechnerisch ermittelt, indem $\varrho r^{2}$ $=$ const. $e^{-r / 20,4}$ gesetzt wurde. Es ergab sich so $B=44,1 \mathrm{~cm}$. Dieser Wert ist wegen der in $\mathrm{Ab}$ schnitt $2 \mathrm{~g}$ behandelten Randstörungen um $0,8 \mathrm{~cm}$ $\mathrm{zu}$ erhöhen. Somit wird die Bremslänge der $\mathrm{Ra}+\mathrm{Be}$-Neutronen für Verlangsamung auf $1 \mathrm{eV}$ in dem untersuchten Beton der Dichte 2,26:

$$
\text { - } B=44,9 \mathrm{~cm} \text {. }
$$

Bei vollständiger Abbremsung auf thermische Energie wird sich dieser Wert um einige cm erhöhen. Der Zuwachs kann wegen des ungenau bekannten H-Gehaltes und der unübersichtlichen Atombindungen im Beton nicht sicher angegeben werden.

b) Abklinglängen. Wie schon Amaldi und Fermi ${ }^{4}$ fanden, fällt in einem großen Wasservolumen die Dichte @ von thermischen Neutronen und auch von überthermischen Resonanzneutronen in größeren Abständen $r$ von einer $\mathrm{Ra}+\mathrm{Be}$ Quelle annähernd nach dem Gesetz ab:

$$
\varrho r^{2}=\text { const } \cdot e^{-r / L^{\prime}} .
$$

8 Ein mit $10 \%$ Wasser angesetzter Probewürfel von $10 \mathrm{~cm}$ Kantenlänge, der also wegen seiner relativ großen Oberfläche stark austrocknete, verlor bis zur Erreichung der Gewichtskonstanz genau die Hälfte seines Wassergehaltes.
Sie fanden die „Abklinglänge“ $L^{\prime}$ in Wasser für thermische und Resonanzneutronen gleich $9,4 \mathrm{~cm}$.

Hier sollten nun Abklinglängen im Beton bestimmt werden, vorausgesetzt, daß für diesen ein gleiches einfaches Grenzgesetz wie für Wasser gilt.

Die Abklinglänge der 1-eV-Neutronen ergibt sich sofort aus der unter a) beschriebenen Messung mit den In-Sonden. Die Kurve $D$ in Abb. 1 zeigt, daß der Abfall von o $r^{2}$ für die 1-eV-Neutronen in einem großen Bereich tatsächlich durch eine $e$-Funktion darstellbar ist. Die in Abb. 1 an-

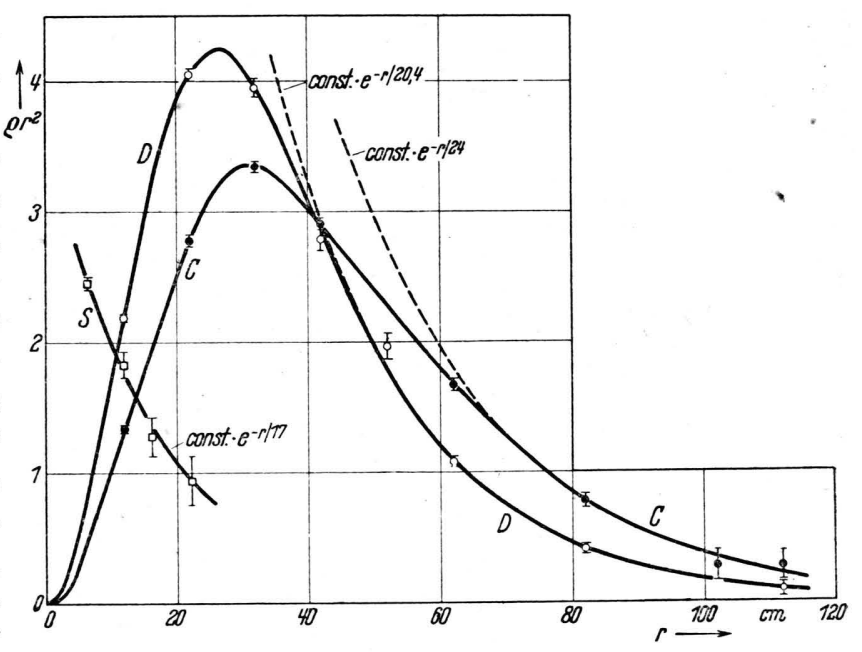

Abb. 1. Verteilung von thermischen Neutronen (Kurve $C$ ), von In-Resonanzneutronen (Kurve D) und von schnellen Ra+Be-Neutronen mit Energien über 3 eMV (Kurve $S$ ) in Beton. $r$ Abstand von der $\mathrm{Ra}+\mathrm{Be}-\mathrm{Neu}-$ tronenquelle, @ Neutronendichte.

gegebene Abklinglänge von 20,4 cm ist wegen der Randstörungen noch etwas $\mathrm{zu}$ erhöhen (vergl. Abschnift $2 \mathrm{~g}$ ). Im unbegrenzten Betonvolumen wäre die Abklinglänge der $1-\mathrm{eV}$-Neutronen $L^{\prime}=21,8 \mathrm{~cm}$.

Ferner wurde der Dichteverlauf der thermischen Neutronen im Beton in derselben Anordnung, aber mit dünnen Dysprosiumsonden gemessen. Das Ergebnis ist ebenfalls in Abb. 1, Kurve $C$, dargestellt; diese Kurve ist auf denselben Flächeninhalt gebracht wie Kurve $D$. Offenbar ist der Abfall von $\varrho r^{2}$ für die thermischen Neutronen in dem untersuchten Bereich weniger gut durch eine $e$ Funktion darzustellen. Die Abklinglänge für den Ausläufer der Kurve ist etwa $24 \mathrm{~cm}$; die Korrektion wegen Randstörungen kann für diesen Wert vernachlässigt werden. Es scheint, daß der 
Abfall der thermischen Neutronen für große $r$ in den Abfall der 1-eV-Neutronen übergeht.

Endlich wurde noch der Dichteverlauf derjenigen schnellen $\mathrm{Ra}+\mathrm{Be}-\mathrm{Neutronen}$ gemessen, die Aluminium gemäß dem Schema $\mathrm{Al}^{27}(n, p) \mathrm{Mg}^{27}$ aktivieren. Die Energietönung dieser Reaktion ist $-2 \mathrm{eMV}^{9}$, ferner braucht das Proton mindestens eine Energie von etwa $1 \mathrm{eMV}$, um mit einiger Wahrscheinlichkeit den $\mathrm{Al}^{28-Z}$ wischenkern verlassen zu können; auch experimentell hat $\mathrm{Hill}^{10}$ die Schwellenenergie der Reaktion zu etwa 3 eMV bestimmt. Daher werden durch die 10-min-Aktivität des $\mathrm{Mg}^{27}$ nur Neutronen von mindestens $3 \mathrm{eMV}$ nachgewiesen, d. h. also nur die schnellsten Neutronen des Ra+Be-Spektrums, die kaum Zusammenstöße erlitten haben.

Bei diesem Versuch wurden $2 \mathrm{~mm}$ dicke AlSonden verwendet, die mit $1 \mathrm{~mm}$ starkem Cd gegen thermische Neutronen abgeschirmt waren. Trotzdem trat die 2,3-min-Aktivität des $\mathrm{Al}^{28}$ auf ${ }^{\mathbf{1 1}}$, mit einer Anfangsaktivität von derselben Größenordnung wie die des gewünschten $\mathrm{Mg}^{27}, T=10 \mathrm{~min}$. Außerdem entstand noch sehr schwach $\mathrm{Na}^{24}$, $T=15 \mathrm{~h}$, dessen Beitrag nachträglich festgestellt und abgezogen wurde. Um die Anfangsaktivität des $\mathrm{Mg}^{27}$ möglichst genau $\mathrm{zu}$ erhalten, wurde nicht das Abklingen der 2,3-min-Periode abgewartet, sondern es wurde der komplexe Abfall der beiden Aktivitäten ausgewertet. Störende Aktivitäten von Verunreinigungen des Aluminiums oder dergl. waren nicht merklich vorhanden.

Die so bestimmten Anfangsaktivitäten des $\mathrm{Mg}^{27}$ sind ein Maß für eine mittlere Dichte der schnellen $\mathrm{Ra}+\mathrm{Be}$-Neutronen mit Energien über $3 \mathrm{eMV}$ im Beton. Die Dichtewerte sind, multipliziert mit dem Quadrat des Quellabstandes $r$, ebenfalls in Abb. 1 eingetragen (Kurve $S$ ). Der Abstand $r$ ist dảbei vom Zentrum der kugelförmigen $\mathrm{Ra}+\mathrm{Be}$ Quelle von 2,2 cm $\varnothing$ bis zur Mitte der Sonden gerechnet. Wie man sieht, liegen die Meßpunkte auf einer Exponentialkurve mit der Abklinglänge . $17 \mathrm{~cm}$.

c) Abdämmung von Neutronendurch eine Betonwand. Direkte Angaben über die

9 Nach W. Gentner u. H. Maier-Leibnitz (unveröffentlicht) ist die im Isotopenbericht 1942 von Flügge u. Matta u ch (Physik. Z. 44, 181 [1943]) angegebene Masse von $\mathrm{Mg}^{27}$ um 1 TME zu erhöhen, da alle $\beta$-Strahlen von $\mathrm{Mg}^{27}$ mit $\gamma$-Strahlung gekoppelt sind. Mit dieser größeren Masse ergibt sich die obige Energietönung.

10 J. E. Hill, Physic. Rev. 57, 1076 [1940].
Schutzwirkung einer Betonwand gegenüber Neutronen sind durch folgenden Versuch zu gewinnen: Auf der einen Seite einer sehr ausgedehnten, freistehenden Betonwand von veränderlicher Dicke $x$ befindet sich die Quelle $Q$ der schnellen Neutronen im Punkte $x=y=0$, s. Abb. 2. Man mißt den Gesamtstrom $I$ der thermischen Neutronen, die zur anderen Seite der Wand (Ebene $x=$ const.) austreten, indem man längs des zur $y$-Achse parallelen Strahls $O P$ die Stromdichte $j$ mißt und das Integral

$$
I(x)=2 \pi \int_{0}^{\infty} j y d y
$$

bildet. Da alle schnelleren Neutronen ja mindestens ebenso rasch abklingen wie die thermischen, genügt es hier, allein das Verhalten der letzteren zu untersuchen.

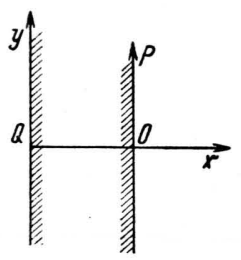

Abb. 2. Zur Messung des Neutronenstromes aus einer Wand.

Zur praktischen Durchführung des Versuchs wurde die obige ideale Anordnung in zwei Punkten verlassen. Erstens wurden die $\mathrm{Ra}+\mathrm{Be}-\mathrm{Neutronen}$ durch Paraffin vorgebremst; die Neutronenquelle befand sich im Zentrum eines Paraffinzylinders von $20 \mathrm{~cm} \varnothing$ und $18 \mathrm{~cm}$ Höhe. Es trat also ein Gemisch von schnellen und langsamen Neutronen in die Wand ein. Der Verlauf des Stromes $I(x)$ wurde dadurch für kleine $x$ natürlich stark verändert; dagegen ist nicht anzunehmen, daß die Steilheit des für größere Wandstärken zu erwartenden quasi exponentiellen Abfalls von I, die hier hauptsächlich interessierte, durch das Paraffin merklich geändert wurde. Dieser Abfall stellte sich aber schon bei geringeren Betondicken ein, und dies hatte den Vorteil, daß die Abmessungen der Betonwand in erträglichen Grenzen gehalten

11 Nach F. Rasetti (Physic. Rev. 58, 869 [1940]) ist in Paraffin die 2,3-min-Resonanzaktivität etwa gleich $2 \%$ der thermischen Aktivität. Jedoch steht wohl noch nicht fest, ob die fragliche Aktivierung durch eigentlichen Resonanzeinfang bei höherer Energie zustande kommt oder durch den vom Cd durchgelassenen Ausläufer der thermischen Verteilung bewirkt wird. 
werden konnten und die Neutronenintensität nicht zu klein wurde.

Zweitens wurde die obige Wandanordnung insofern verlassen, als die Wand nicht freistehend errichtet, sondern in einem normalen Meßzimmer

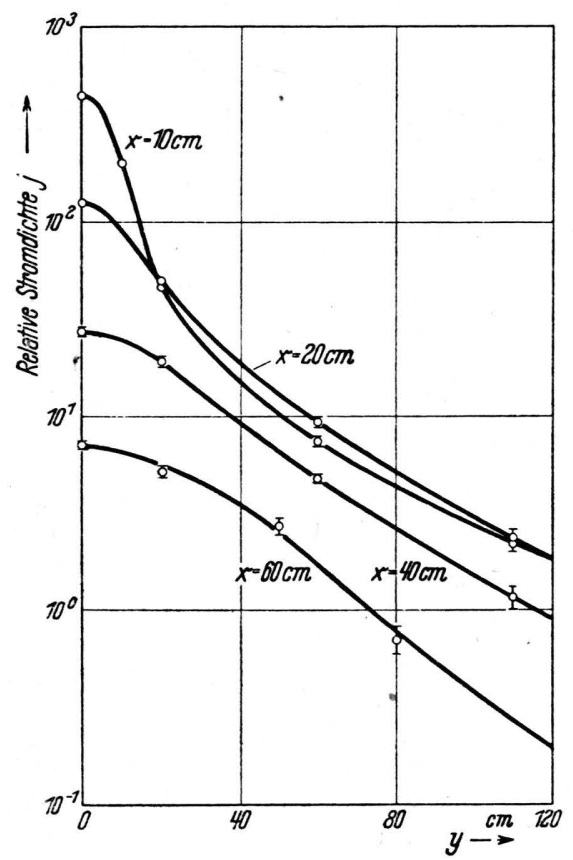

Abb. 3. Dichte $j$ des thermischen Neutronenstromes aus Betonwänden. $x$ Wandstärke, $y$ Abstand von der Wandnormalen durch die Ra+Be-Quelle.

in $20 \mathrm{~cm}$ Höhe über dem Fußboden flachliegend angeordnet wurde. Die Fläche der Wand betrug $1,5 \times 2 \mathrm{~m}^{2}$, ihre Dicke 10 bis $60 \mathrm{~cm}$, sie ruhte auf 10 kleinen $20 \mathrm{~cm}$ hohen Betonklötzen. Die Neutronenquelle befand sich zwischen Fußboden und Wand in $55 \mathrm{~cm}$ Abstand von der einen Schmalseite und $75 \mathrm{~cm}$ Abstand von den Längsseiten der Wand. Durch den sehr nahen Fußboden und auch durch die Decke und Wände des Zimmers wurde die Neutronenverteilung in der Versuchswand natürlich stark und in unübersichtlicher Weise beeinflußt. Diese Störungen wurden, soweit sie durch Rückstreuung thermischer Neutronen bedingt waren, dadurch ausgeschaltet, daß die Betonwand beiderseitig mit $0,5 \mathrm{~mm}$ starkem Cd-Blech belegt wurde, außer an der Stelle der Neutronenquelle. Daneben blieb noch die Möglichkeit, daß überthermische Neutronen aus der Umgebung in die Wand zurückgestreut, dort thermisch wurden und zu dem austretenden Strom einen Beitrag lieferten. Eine einfache Utberlegung zeigt jedoch, daß für die größeren Wandstärken von 40 bis $60 \mathrm{~cm}$ diese Neutronen die Steilheit des Stromabfalls nicht beeinflussen.

Die Stromdichte $j$ der thermischen Neutronen wurde mit dicken Dy-Sonden gemessen, die unter die obere Cd-Belegung gelegt wurden.

Abb. 3 zeigt $j$ in logarithmischem Maßstab. Für vier Wandstärken, $x=10,20,40$ und $60 \mathrm{~cm}$, wurden die Kurven $j(y)$ aufgenommen. Nach diesen ausgeglichenen Kurven wurden nun die Kurven für $j \cdot y$ gezeichnet, und aus ihnen wurden gemäß Gl. (3) durch Planimetrieren die austretenden Gesamtströme $I$ gewonnen, die in Abb. 4 in $\mathrm{Ab}$ hängigkeit von der Wandstärke $x$ in logarithmischem Maßstab dargestellt sind. Die Teilintegrale von der Grenze des untersuchten $y$-Bereiches bis $\infty$ wurden dabei rechnerisch bestimmt, wozu die $j y$-Kurven exponentiell extrapoliert wurden. Legt man nun in Abb. 4 durch die Meßpunkte bei $x=40$ und $x=60 \mathrm{~cm}$ eine logarithmische Gerade, so erhält man als deren Abklinglänge 15,3 cm. In dem Falle, daß die Neutronenquelle nicht mit Paraffin umgeben ist, darf nach dem obigen dieselbe Abklingung, jedoch erst bei größeren Wandstärken, angenommen werden. Der ungefähr horizontale Anfangsteil der $I$-Kurve ist durch das Paraffin bedingt; ohne das Paraffin müßte der thermische Neutronenstrom zunächst ansteigen.

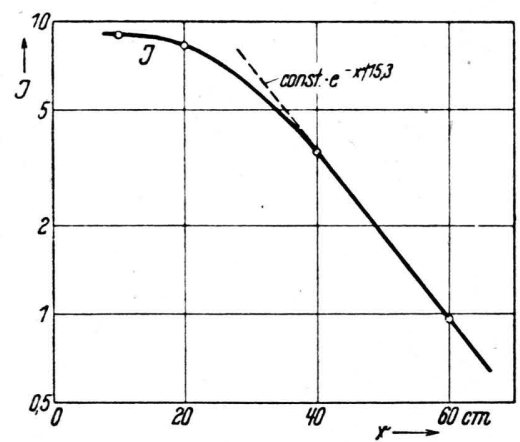

Abb. 4. Gesamtstrom $I$ der thermischen Neutronen aus Betonwänden verschiedener Dicken $x$.

\section{Diskus sion}

a) Berechnung der Bremslänge aus den Wirkungsquerschnitten. Die Dichte des Betons betrug $2,26 \mathrm{~g} / \mathrm{cm}^{3}$. Sein Wassergehalt wird mangels genauerer Kenntnis in den folgenden Rechnungen einmal zu 5 und einmal zu 10 Gewichts - \% angenommen (vergl. Abschn. 1a). Der Zementgehalt betrug $15 \%$; die restlichen $80 \%$ 
bzw. 75\% waren Kiessand. Als Bestandteile des Zementes werden angenommen ${ }^{12}: 65 \% \mathrm{CaO}, 25 \%$ $\mathrm{SiO}_{2}, 7 \% \mathrm{Al}_{2} \mathrm{O}_{3}, 3 \% \mathrm{Fe}_{2} \mathrm{O}_{3}$; sonstige Beimengungen wie $\mathrm{MgO}$ usw. werden vernachlässigt. Der Kiessand wird als reines $\mathrm{SiO}_{2}$ behandelt. Damit ergeben sich die in Tab.1, Spalte 2 angegebenen Atomzahlen $N$ im $\mathrm{cm}^{3}$; bei $\mathrm{H}, \mathrm{O}$ und $\mathrm{Si}$ beziehen sich die oberen Zahlen auf 5, die unteren auf $10 \%$ Wassergehalt des Betons.

Die zur Berechnung ferner benötigten mittleren Streuquerschnitte $\sigma$ (in Spalte 3 der Tab. 1) werden für $\mathrm{H}$ und $\mathrm{O}$ der früheren Arbeit ${ }^{7}$ entnommen, für die übrigen Kerne werden plausible Mittelwerte angenommen ${ }^{13}$.

Mit diesen Daten ergibt sich nach den bei $\mathrm{B}$ othe $\mathrm{e}^{5}$ und $\mathrm{Jensen} \mathrm{n}^{7}$ angegebenen Formeln für eine mittlere Primärenergie von $3 \mathrm{eMV}$ und die Endenergie $1 \mathrm{eV}$ die Bremslänge im Beton $\mathrm{zu}$ $57,3 \mathrm{~cm}$ für $5 \%$ Wassergehalt und $\mathrm{zu} 41,0 \mathrm{~cm}$ für $10 \%$ Wassergehalt. Der gemessene Wert von $44,9 \mathrm{~cm}$ liegt dazwischen.

Aus der nahen Übereinstimmung zwischen dem gemessenen und dem für $10 \%$ Wassergehalt berechneten $B$-Wert ist noch nicht zu schließen, daß der Wassergehalt des Betons näher bei $10 \%$ lag. Dazu sind erstens die Wirkungsquerschnitte und die Bestandteile des Betons nicht genau genug bekannt, und zweitens könnten im Beton vielleicht auch unelastische Stöße zur Bremsung beitragen.

b) Die Streuweglängeder primären schnellen Neutronen und die $A_{0} b-$ $\mathrm{kl}$ lingläng en. Verschiedentlich ist die Ansicht geäußert worden, daß die Abklinglänge langsamer Neutronen (vergl. Abschn. 1b) mit der Streuweglänge $\lambda_{0}$ der primären schnellen Neutronen übereinstimmen müsse, sofern nur die Streuweglänge mit der Energie abnimmt. Nach der Theorie von Bothe ${ }^{5}$ ist dies Verhalten jedoch für solche Quellabstände $r$, in denen die Dichte der langsamen Neutronen, o, noch praktisch meßbar ist, nicht $\mathrm{zu}$ erwarten. Wenn in diesem Bereich doch ein quasiexponentieller Abfall von $\mathrm{o} r^{2}$ beobachtet wird, wie z.B. beim Wasser, so ist dies nicht als Folge eines strengen Gleichgewichtes zwischen primären und langsamen Neutronen anzusehen, sondern es hat andere Gründe. Tatsächlich ist die beobachtete Abklinglänge im Wasser auch deutlich größer als $\lambda_{0}$.

12 Vergl. z. B. „Hütte“, Bd. I.

13 Nach d. Zusammenstellung v. K. Di ebner, W. Herrmann u. E. Graßmann, Physik. Z. 43, 440 [1942].

\begin{tabular}{|c|c|c|c|c|c|}
\hline 1 & 2 & 3 & 4 & 5 & 6 \\
\hline $\begin{array}{l}\text { Ele- } \\
\text { ment }\end{array}$ & $\begin{array}{c}N \\
10^{21} / \mathrm{cm}^{3}\end{array}$ & $\begin{array}{c}\sigma \\
10^{-24} \\
\mathrm{~cm}^{2}\end{array}$ & $\begin{array}{c}\sigma_{s} \\
(3 \mathrm{eMV}) \\
10^{-24} \\
\mathrm{~cm}^{2}\end{array}$ & $\begin{array}{c}\sigma_{s} \\
\text { (therm.) } \\
10^{-24} \\
\mathrm{~cm}^{2}\end{array}$ & $\begin{array}{c}\sigma_{a} \\
\text { (therm.) } \\
10^{-24} \\
\mathrm{~cm}^{2}\end{array}$ \\
\hline $\mathrm{H}$ & $15^{7,6}$ & 5,27 & 2,0 & 45 & 0,25 \\
\hline 0 & $\begin{array}{l}45 \\
46\end{array}$ & 2,2 & 1,2 & 4,1 & 0 \\
\hline $\mathrm{Si}$ & $\begin{array}{l}19 \\
18\end{array}$ & 3,0 & 2,5 & 2,8 & 0,01 \\
\hline $\mathrm{Al}$ & 0,28 & 2,5 & 2,3 & 1,6 & 0,43 \\
\hline $\begin{array}{l}\mathrm{Ca} \\
\mathrm{Fe}\end{array}$ & $\begin{array}{l}2,4 \\
0,075\end{array}$ & 5 & $\begin{array}{l}3,7 \\
3\end{array}$ & $\begin{array}{l}10 \\
10\end{array}$ & $\begin{array}{l}0,4 \\
2\end{array}$ \\
\hline
\end{tabular}

Tab. 1. Atomzahlen und Wirkungsquerschnitte.

Dasselbe geht aus den vorliegenden Messungen am Beton noch klarer hervor. Legt man die in Spalte 4 der Tab. 1 angegebenen Streuquerschnitte, die für eine mittlere Neutronenenergie von $3 \mathrm{eMV}$ angenommen sind ${ }^{13}$, zugrunde, so erhält man für den Beton mit 5 bis $10 \%$ Wassergehalt $\lambda_{0}=7$ bis $8 \mathrm{~cm}$. Selbst wenn dieser Wert wegen der Unsicherheit der benutzten Wirkungsquerschnitte noch etwas zu erhöhen wäre, so ist er doch viel kleiner als die gemessenen Abklinglängen für die langsamen und auch für die schnellen Neutronen (22 bis $24 \mathrm{~cm}$, bzw. $17 \mathrm{~cm}$ ). Für die schnellen Neutronen wäre offenbar nur dann die gemessene Abklinglänge gleich $\lambda_{0}$, wenn die Energie eines jeden Neutrons gleich beim ersten Stoß unter die Nachweisschwelle sänke, was bei unseren Messungen sicher nicht der Fall war.

c) Die Diffusionslänge der thermischen Neutronen und die Abklinglängen. Aus dem verschiedenen Charakter der Kurven $C$ und $D$ in Abb. 1 geht hervor, daß die Neutronen im Beton auch nach der Abbremsung noch merklich auseinanderdiffundieren. Tatsächlich sind auch beim Beton die Abklinglänge und die thermische Diffusionslänge nicht so verschieden wie z. B. beim Wasser. In den Spalten 5 und 6 der Tab. 1 sind die thermischen Streu- und Absorptionsquerschnitte der im Beton enthaltenen Kerne angegeben ${ }^{14}$ (die Absorptionsquerschnitte der hauptsächlich vorhandenen Elemente O und Si

${ }^{14}$ Nach ${ }^{13}$ und der neueren Zusammenstellung bei H. Volz, Z. Physik 121, 201 [1943]; die in Tab. 2, Spalte III dieser Arbeit angegebenen Werte für Si sind verdruckt. 
sind allerdings nur der Größenordnung nach bekannt). Damit ergibt sich für 5 bzw. 10\% Wassergehalt die Streuweglänge $\lambda_{s}$ zu 1,7 bzw. $1,1 \mathrm{~cm}$, und die Absorptionsweglänge $\lambda_{a} \mathrm{zu} 300 \mathrm{bzw}$. $190 \mathrm{~cm}$. Die nach der Fermischen Formel $l^{2}=\frac{1}{3} \lambda_{s} \lambda_{a}$ resultierende Diffusionslänge $l$ von $13 \mathrm{bzw} .8,3 \mathrm{~cm}$ ist also rund halb so groß wie die Abklinglänge $L^{\prime}$.

Beim $\mathrm{H}_{2} \mathrm{O}$ ist die weitere Ausbreitung der Neutronen nach der Verlangsamung im allgemeinen $\mathrm{zu}$ vernachlässigen; hier ist das Verhältnis von Abkling- zu Diffusionslänge etwa 4. Um beim Beton dasselbe Verhältnis herzustellen, hätte man dem Beton mit $5 \%$ Wassergehalt z. B. $3,8 \cdot 10^{19}$ Boratome pro $\mathrm{cm}^{3}$, d. h. 0,68 kg Bor pro Kubikmeter Beton zuzusetzen.

d) Berechnung der Abklinglänge für langsame Neutronen. Man kann die Verlangsamung eines Neutrons von der Primärenergie $E_{0}$ auf die Energie $E$ als einen mit $A b$ sorption verbundenen Diffusionsprozeß ansehen in dem Sinne, daß ein schnelles Neutron als solches absorbiert ist, wenn seine Energie unter $E$ gesunken ist. Bei hinreichend großer mittlerer Stoßzahl $n$ kann man den Wirkungsquerschnitt dieser „Absorption“ gleich $\sigma_{s} / n$ setzen, wenn $\sigma_{s}$ den Streuquerschnitt bedeutet, denn die Absorption findet im Mittel nach Zurücklegung von $n$ freien Weglängen statt. Eine mit diesem Absorptionsquerschnitt nach der Fermischen Formel berechnete Diffussionslänge wird Aufschluß über die räumliche Verteilung derjenigen Neutronen geben, deren Energie größer als $E$ ist.

Um aus dieser Auffassung quantitative Schlüsse auf die Abklinglänge langsamer Neutronen zu ziehen, legen wir dasselbe vereinfachte Brems. schema zugrunde wie in den früheren Arbeiten ${ }^{5,7}$, d.h. wir nehmen an, daß der einzelne Streuprozeß isotrop verläuft und daß das Neutron in einem elementaren Stoff bei jedem Stoß auf denselben Bruchteil 1/q seiner Energie abgebremst wird, wo

$$
q=\frac{(M+1)^{2}}{M^{2}+1}
$$

( $M=$ Massenzahl des streuenden Kerns). Die zur Abbremsung notwendige Stoßzahl ist dann

$$
n=\frac{\log \left(E_{0} / E\right)}{\log q} .
$$

Ferner nehmen wir hier an, der Streuquerschnitt sei unabhängig von der Energie und gleich dem mittleren effekten Streuquerschnitt $\sigma$, der in der früher abgeleiteten Formel

$$
B^{2}=\frac{2 n}{(N \sigma)^{2}}
$$

für das Bremslängenquadrat auftritt ( $N=$ Atom$\left.\mathrm{zahl} / \mathrm{cm}^{3}\right)$. Mit diesem Streuquerschnitt

$$
\sigma=\frac{\sqrt{2 n}}{N B}
$$

und dem Absorptionsquerschnitt

$$
\sigma_{a}=\sigma / n=\frac{\sqrt{2 / n}}{N B}
$$

ergibt sich für die nach der üblichen Vorschrift gebildete „Diffusionslänge“ $L$ :

$$
L^{2}=\frac{1}{3 N^{2} \sigma \sigma_{a}}=\frac{1}{6} B^{2}=\frac{n}{3(N \sigma)^{2}} .
$$

Bei mehratomigen Stoffen bleibt die Beziehung $L=B / \sqrt{6}$ bestehen ${ }^{15}$, wobei für $B$ der früher mitgeteilte allgemeine Ausdruck einzusetzen ist ${ }^{5,7}$.

Diese Länge $L$ sollte, wenn das zugrunde gelegte Bremsschema zur Wiedergabe der Wirklichkeit geeignet ist, den Dichteabfall der ,schnellen“ Neutronen, d.h. aller Neutronen mit Energien $>E$, regeln. Nach der Diffusionsgleichung sollte z.B. die Dichte o dieser Neutronen bei Voraussetzung einer punktförmigen Quelle schneller Neutronen im unbegrenzten homogenen Mittel nach dem Gesetz

$$
\varrho r=\mathrm{const} \cdot e^{-r / L}
$$

abfallen. In größeren Abständen von der Quelle, wo annähernd ein Gleichgewicht zwischen den Neutronen aller Geschwindigkeiten besteht, sollte auch die Dichte der langsamen Neutronen nach diesem Gesetz abfallen. Bei Wasser ist dies in der Tat für $r>10 \mathrm{~cm}$ erfüllt, wie man z. B. den Messungen von Amaldi und $\mathrm{Fermi}^{4}$ entnehmen kann, oder wie es aus neueren Messungen von v. Droste ${ }^{16}$ unmittelbar hervorgeht, der die thermische o $r$-Kurve bis $r=55 \mathrm{~cm}$ aufgetragen hat. Die Abklinglänge der @r-Kurve für Wasser ist danach etwa $7 \mathrm{~cm}$, und dies ergibt, mit $\sqrt{ } 6 \mathrm{multi}-$ pliziert, tatsächlich die von A maldi und Fermi gemessene Bremslänge des Wassers von $17 \mathrm{~cm}$.

15 F. B op p (unveröffentlicht) hat unahhängig von uns dieselbe Beziehung aus ähnlichen Überlegungen abgeleitet.

16 G. v. D r os te, unveröffentlicht. 
Man ersieht hieraus auch, daß im kugelsymmetrischen Falle nicht die vielfach diskutierte $\varrho r^{2-}$ Kurve, sondern die or-Kurve eine einfache Bedeutung hat. Der beste Beweis dafür ist, daß experimentell die Abklinglänge der o $r$-Kurve übereinstimmt mit der Abklinglänge von $\varrho$ im ebenen Fall (vergl. Abschn. 2e).

Allerdings läßt sich in ähnlicher Annäherung wie $\varrho r$ auch die Größe $\varrho r^{2}$ in größeren Bereichen durch eine Exponentialfunktion ersetzen. Zwischen der Abklinglänge $L^{\prime}$ von $\varrho r^{2}$ und der Abklinglänge $L$ von $\varrho r$ besteht offenbar die einfache Beziehung

$$
\frac{1}{L^{\prime}}=\frac{1}{L}-\frac{1}{r}
$$

Prüft man die obigen Näherungsformeln nun an den Meßergebnissen für Beton, so findet man folgendes: Die gemessene Bremslänge für die Endenergie $1 \mathrm{eV}$ war $44,9 \mathrm{~cm}$. Hieraus folgt nach Gl. (8) $L=44,9 / \sqrt{6}=18,3 \mathrm{~cm}$, und als Abkling länge $L^{\prime}$ des $\varrho r^{2}$-Abfalls ergibt sich damit für ein mittleres $r$ von $80 \mathrm{~cm}$ nach Gl. (10) der Wert $L^{\prime}=23,7 \mathrm{~cm}$. In guter Übereinstimmung hiermit wurde als Abklinglänge der $1-\mathrm{eV}$-Neutronen $L^{\prime}=21,8 \mathrm{~cm}$ direkt gemessen.

Da die Bremslänge sich mit einer für praktische Zwecke ausreichenden Genauigkeit aus den Wirkungsquerschnitten berechnen läßt, gilt dasselbe also auch für die Abklinglänge der langsamen Neutronen.

e) Diffusion von Neutronen durch e ine Wand. Nach Fermi ist die Wahrscheinlichkeit dafür, daß ein thermisches Neutron, das im Innern eines großen streuenden und absorbierenden Körpers bei $x=0$ startet, zu der Grenzebene $x=$ const. des Körpers austritt, für $x \gg l$ proportional $\mathrm{zu}$

$$
p(x)=e^{-x / 2}
$$

wobei $l$ die Diffusionslänge des thermischen Neutrons in dem betreffenden Stoff ist. Hiermit gleichbedeutend ist die Aussage, daß der von einer thermischen Neutronenquelle herrührende, durch die Grenzebene $x=$ const. austretende Gesamtstrom dieselbe Abhängigkeit von $x$ aufweist. Grenzt der betrachtete Körper auch in der Ebene $x=0$ ans Vakuum, so überlegt man sich leicht (vergl. die Arbeit von Jense n ${ }^{7}$, Abschn. 2), daß der Strom durch die Ebene $x$ nun proportional $\mathrm{zu} d p / d x$ ist, also wiederum wie $e^{-x / l}$ abfällt.
Dieses Ergebnis läßt sich sofort auf den Fall übertragen, daß die Quelle schnelle Neutronen emittiert, wenn man wieder statt $l$ die in Abschn. $2 \mathrm{~d}$ eingeführte „Diffusionslänge $L$ der schnellen Neutronen" einsetzt. Für genügende Wanddicken $x$, in denen sich annähernd ein Gleichgewicht zwischen schnellen und langsamen Neutronen einstellen kann, sollte danach der Gesamtstrom der austretenden langsamen Neutronen wie $e^{-x / L}$ abfallen.

Die in Abschn. 1c beschriebenen Messungen an der Versuchswand erlauben, diese Aussage für Beton zu prüfen. Es ergab sich dort die Abklinglänge des thermischen Stromes $I \mathrm{zu} L=15,3 \mathrm{~cm}$ (vergl. Abb.4). Andererseits ist nach Abschn. $1 \mathrm{~b}$ die Abklinglänge $L^{\prime}$ der Größe $\varrho r^{2}$ der thermischen Neutronen 22 bis $24 \mathrm{~cm}$. Nach Gl. (10) ist dann für ein mittleres $r$ von $80 \mathrm{~cm} L=17,3$ bis $18,5 \mathrm{~cm}$. Die Ubereinstimmung der beiden $L$-Werte ist als befriedigend $\mathrm{zu}$ bezeichnen, wenn man die geringe Genauigkeit der Strommessung bei $x=60 \mathrm{~cm}$ berücksichtigt (vergl. Abb. 3).

Auch bei der Anwendung auf $\mathrm{H}_{2} \mathrm{O}$ bewährt sich die obige Überlegung. Z. B. nimmt nach einer Messung von Riezle ${ }^{17}$ an einer dicken Wasserwand die Dichte der langsamen Neutronen (und dasselbe muß für den Strom gelten) senkrecht zur Wand ungefähr exponentiell ab mit einer Halbwertlänge von $5 \mathrm{~cm}$; d. h. $L=7 \mathrm{~cm}$ in Übereinstimmung mit Abschn. 2d.

f) Praktische Bemessung von Neutronenschutzwänden. Nach einem Artikel von $\mathrm{B}$ othe $\mathrm{e}^{18}$ über den Strahlenschutz bei kernphysikalischen Untersuchungen kann man für die erforderliche Dicke $D$ einer Neutronenschutzwand ansetzen:

$$
D \approx L \cdot \log k+B,
$$

wobei $L$ die Abklinglänge der thermischen Neutronendichte (oder auch des Stromes) senkrecht zur Wand, 1/k der hinter der Wand zugelassene Bruchteil der Primärintensität und $B$ die Bremslänge ist. $B$ ist als ,Vorschaltdicke" anzusehen, von der ab der quasi-exponentielle Abfall der Intensität stattfindet. Für (12) kann man nun nach (8) auch schreiben

$$
D \approx B(1+\log k / \sqrt{6}) .
$$

Verlangt man eine Schwächung der thermischen Neutronen auf $10^{-3}$ (alle schnelleren Neutronen

17 W. Riezler, unveröffentlicht.

$18 \mathrm{~W}$. B oth e, unveröffentlicht. 
sind dann mindestens ebenso stark vermindert), so ergibt sich für den hier untersuchten Beton mit $L=16 \mathrm{~cm}$ und $B=45 \mathrm{~cm}$

$$
D=160 \mathrm{~cm} .
$$

Diese beträchtliche Dicke läßt sich auch durch Zusatz von Bor kaum vermindern, denn der in Abschn. 1b gefundene Unterschied zwischen den Abklinglängen der thermischen und der fast-thermischen Neutronen ist jedenfalls nur sehr gering.

Entscheidend herabsetzen läßt sich die Wandstärke nur durch Erhöhung des Wasserstoffgehaltes. Eine $\mathrm{H}_{2} \mathrm{O}$-Schicht, die die Neutronen auf $10^{-3}$ schwächt, brauchte nur $65 \mathrm{~cm}$ dick zu sein, jedoch wäre damit die stets auch vorhandene $\gamma$-Strahlung noch nicht genügend reduziert. Es sei erwähnt, daß Rajewsky ${ }^{19}$ Spezialbaustoffe für feste Neutronenschutzwände entwickelt hat, deren $\mathrm{H}$-Gehalt den des normalen Betons erheblich übertrifft.

g) Die Größe des Betonvolumens. Zur Ableitung der Bremslänge und der Abklinglängen der langsamen Neutronen aus den Messungen von Abschn. $1 \mathrm{a}$ und $\mathrm{b}$ wurde angenommen, daß die Dichte @ der langsamen Neutronen auf der Achse des 1/1/2 m großen Betonprismas mit der Dichte $\varrho_{\infty}$ im unbegrenzten Körper praktisch übereinstimmt. Diese Annahme soll hier näher geprüft werden.

Erstens könnte $\varrho$ für große $r$ durch aus dem Zimmer zurückgestreute Neutronen gegenüber $\varrho_{\infty}$ erhöht sein. Eine rohe Abschätzung ergibt jedoch, daß dieser Effekt vernachlässigt werden kann. Zweitens könnte @ gegenüber $\varrho_{\infty}$ dadurch merklich erniedrigt sein, daß Neutronen durch die Oberfläche des Prismas entweichen. Dieser Effekt läßt sich quantitativ erfassen, weil die zunächst nicht bekannte Dichte $\varrho_{\infty}$ aus der gemessenen Dichte $\varrho$ abgeleitet werden kann, wie folgt. Das quadratische Prisma habe die Seitenlänge $a$ und sei hier als unendlich lang vorausgesetzt; seine Achse falle mit der $x$-Achse eines räumlichen rechtwinkligen Koordinatensystems zusammen; die Neutronenquelle befinde sich im Nullpunkt. Wir setzen nun die Dichte $\varrho_{\infty}$ näherungsweise als Summe von Ga u $ß$-Verteilungen an:

$$
\varrho_{\infty}=\sum_{i} c_{i} e^{-\left(r / r_{i}\right)^{2}} .
$$

19 B. Rajewsky, unveröffentlicht.
Dann erhält man für die Dichte @ $(x)$ auf der Prismenachse, wenn man eine schon früher benutzte Úberlegung von $B$ othe hier sinngemäß anwendet (vergl. ${ }^{7}$, Abschn. 2):

$$
\varrho(x)=\sum_{i} c_{i}^{\prime} e^{-\left(x / r_{i}\right)^{2}} .
$$

Dabei ist

$$
\begin{aligned}
c_{i}^{\prime}=c_{i}(1 & -4 e^{-\left(a / r_{i}\right)^{2}}+4 e^{-2\left(a / r_{i}\right)^{2}}+4 e^{-4\left(a / r_{i}\right)^{2}} \\
& \left.-8 e^{-5\left(a / r_{i}\right)^{2}}+4 e^{-8\left(a / r_{i}\right)^{2}}-\ldots\right),
\end{aligned}
$$

wie man sich an Hand von Abb.1 der früheren Arbeit ${ }^{7}$ überlegt. Die $c_{i}^{\prime}$ und $r_{i}$ sind aus der gemessenen Dichteverteilung $\mathbf{Q}(x)$ abzuleiten. Damit kann man nach (15) die $c_{i}$ berechnen und kennt

\begin{tabular}{|c|c|c|c|c|}
\hline \multirow[b]{2}{*}{ Größe } & \multirow[b]{2}{*}{$\begin{array}{l}\text { Neu- } \\
\text { tronen- } \\
\text { energie }\end{array}$} & \multicolumn{3}{|c|}{ Längen in $\mathrm{cm}$} \\
\hline & & $\begin{array}{c}\text { Ge- } \\
\text { messen }\end{array}$ & $\begin{array}{c}\mathrm{Be}- \\
\text { rechnet } \\
\text { für } 5^{\%} \% \\
\mathrm{H}_{2} \mathrm{O}- \\
\text { Gehalt }\end{array}$ & $\begin{array}{c}\mathrm{Be}- \\
\text { rechnet } \\
\text { für } 10 \% \\
\mathrm{H}_{2} \mathrm{O}- \\
\text { Gehalt }\end{array}$ \\
\hline $\begin{array}{l}\text { Bremslänge B } \\
\quad \text { (vgl. Ziff. 1 a } \\
\text { und 2 a) }\end{array}$ & $\begin{array}{c}\mathrm{Ra}+\mathrm{Be} \\
\text { auf } \\
1 \mathrm{eV}\end{array}$ & 45 & 57 & 41 \\
\hline $\begin{array}{l}\text { Abklinglänge } L^{\prime} \\
\text { von } \varrho r^{2}\end{array}$ & $\begin{array}{l}\mathrm{Ra}+\mathrm{Be} \\
>3 \mathrm{eMV}\end{array}$ & 17 & - & - \\
\hline $\begin{array}{l}\text { (vgl. Zitt. 1b } \\
\text { und } 2 \mathrm{~d} \text { ) }\end{array}$ & $\begin{array}{l}1 \mathrm{eV} \\
\text { therm. }\end{array}$ & $\begin{array}{r}22 \\
\sim 24\end{array}$ & $\sim 33$ & $\sim 21$ \\
\hline $\begin{array}{c}\text { A bklinglänge } L \\
\text { d. Neutronen- } \\
\text { stromes durch } \\
\text { eine ebene } \\
\text { Wand (vgl.Ziff. } \\
1 \text { c und 2e) }\end{array}$ & therm. & $\sim 15$ & $\sim 23$ & $\sim 17$ \\
\hline Streuweglänge & $3 \mathrm{eMV}$ & - & 7,9 & 7,1 \\
\hline & therm. & - & 1,7 & 1,1 \\
\hline $\begin{array}{l}\text { Absorptionsweg- } \\
\text { länge }\end{array}$ & therm. & - & 300 & 190 \\
\hline Diffusionslänge & therm. & - & 13 & 8,3 \\
\hline
\end{tabular}
somit $\varrho_{\infty}$.

Wir wollen dies Ergebnis anwenden, um die relative Abweichung $\delta=1-\varrho / \varrho_{\infty}$ für die gemessene Dichteverteilung der In-Resonanzneutronen

Tab. 2. Bremslänge, Abklinglänge, Streuweglänge, Absorptionsweglänge und Diffusionslänge für schnelle und langsame Neutronen in Beton. 
abzuschätzen. Diese Verteilung läßt sich bereits gut durch den zweigliedrigen Ausdruck

$$
\varrho(x)=e^{-(x / 26)^{2}}+0,0333 e^{-(x / 54)^{2}}
$$

approximieren (die Konstante $c_{0}{ }^{\prime}$ ist gleich 1 gesetzt). Mit den nach (15) berechneten $c_{i}(a=100 \mathrm{~cm})$ ist dann

$$
\varrho_{\infty}(r)=e^{-(r / 26)^{2}}+0,0381 e^{-(r / 54)^{2}} .
$$

Danach hat $\delta$ z. B. für die Quellabstände 0, 30, 60, $100 \mathrm{~cm}$ die folgenden Werte: $\delta(0)=0,5 \%, \delta(30)$ $=1,2 \%, \delta(60)=8,7 \%, \delta(100)=12,5 \%$. Da unser Betonprisma auf dem Fußboden lag, der ebenfalls aus Beton bestand, rechnen wir mit $3 / 4$ dieser $\delta$ Werte. Dann ergibt sich, daß die aus der gemessenen $\varrho(x)$-Kurve abgeleitete Abklinglänge der 1-eV-Neutronen um 1,4 cm, die Bremslänge um $0,8 \mathrm{~cm} z u$ erhöhen ist, wie in Abschn. 1 geschehen.

Zusammenfassung. Das Verhalten von schnellen und langsamen Neutronen im Innern von
4,5 Tonnen Beton und beim Durchdringen einer Betonwand variabler Dicke wurde untersucht. Die Dichte des Betons betrug 2,26 g/ $\mathrm{cm}^{3}$, sein Wassergehalt 5 bis 10 Gewichtsprozent. Die gemessenen und berechneten Werte der interessierenden Größen sind in Tab. 2 zusammengestellt; @ ist die Neutronendichte im Abstande $r$ von einer Punktquelle.

Theoretisch werden die einfachen Beziehungen $L \approx B / \sqrt{6}, 1 / L^{\prime}=1 / L-1 / r$ abgeleitet und experimentell bestätigt (Abschn.2d).

$L$ läßt sich auch bei kugelsymmetrischer Anordnung messen, und zwar als Abklinglänge von or (nicht $\varrho r^{2}$ ).

Damit kann außer den Abklinglängen auch die Dicke von Neutronenschutżänden näherungsweise berechnet werden. Eine Schutzwand aus dem hier untersuchten Beton, die die Intensität der Neutronen auf 1/1000 schwächt, müßte $160 \mathrm{~cm}$ dick sein.

Die vorliegenden Messungen wurden auf Veranlassung von Hrn. Prof. B othe ausgeführt. Wir danken ihm für eingehende Diskussionen.

\title{
Eine Fehlermöglichkeit bei massenspektrographischen Dublettmessungen
}

\author{
Von Heinz Ewald \\ Aus dem Kaiser-Wilhelm-Institut für Chemie, Tailfingen \\ (Z. Naturforschg. 2a, 384-388 [1947]; eingegangen am 24. April 1947)
}

\begin{abstract}
Aus massenspektrographischen Aufnahmen wird geschlossen, daß der Durchmesser der Ionenbündel der Kanalstrahlentladung am Ort des Spektrographenspaltes für die verschiedenen gleichzeitig erzeugten Ionensorten sehr verschiedene Werte annimmt. So haben z. B. die O+-Bündel einen Durchmesser von Millimetern, während die $\mathrm{CH}_{4}^{+-}$ Bündel auf nur einige Zehntel-Millimeter zusammengedrängt sind, den Spalt also nur auf einem geringen Teil seiner Länge ausleuchten. Bei idealer Justierung des Spaltes senkrecht zu den Feldlinien des elektrischen Ablenkfeldes sollte diese verschiedenartige Ausleuchtung auf die Messungen ohne Einfluß bleiben. Aber schon bei einer Verdrehung des Spaltes von nur $0,1^{\circ}$ um die Richtung der Ionenstrahlen können hierdurch unter gewissen Umständen systematische Fehler entstehen, die die in den neueren Arbeiten angegebenen wahrscheinlichen Fehler der Ergebnisse um ein Mehrfaches übersteigen. Es wird angenommen, daß die immer noch nicht vollkommen befriedigende Übereinstimmung der Resultate der verschiedenen Autoren für das wichtige Dublett ${ }^{12} \mathrm{CH}_{\mathbf{4}}-\mathbf{1 6 O}^{16}$ hierin ihre Erklärung findet.
\end{abstract}

$\mathrm{D}$ ie Frage nach der Genauigkeit und Übereinstimmung der massenspektrographischen $\mathrm{Du}^{-}$ blett-Messungen, insbesondere der sogenannten Grunddubletts, ist in den letzten zehn Jahren mehrfach in kritischen Untersuchungen hervorgetreten. Als Aston 1936 seinen dritten Massenspektrographen fertiggestellt hatte ${ }^{1}$, zeigte sich bei der Bestimmung des Dubletts ${ }^{1} \mathrm{H}_{2}-{ }^{2} \mathrm{D}$ eine Linien- schärfe, die theoretisch eine Genauigkeit der Massenbestimmung von $1: 10^{5}$ hätte ermöglichen sollen. Bei der Auswertung verschiedener Einzelaufnahmen zeigten die Ergebnisse aber untereinander Schwankungen, die zu groß waren, um mit einer solchen Genauigkeit verträglich zu sein. Die Ursache hierfür sah Aston in den wechselnden 1 F. W. Aston, Nature [London] 137, 357 [1936]. 